\title{
Planning monitoring programmes for cyanobacteria and cyanotoxins
}

Martin Welker, Ingrid Chorus, Blake A. Schaeffer and Erin Urquhart

\section{CONTENTS}

Introduction and general considerations 641

$\begin{array}{ll}11.1 & \text { Designing a monitoring programme }\end{array} 644$

11.1.1 Collecting and analysing existing knowledge 644

11.1.2 Defining the objective of monitoring 646

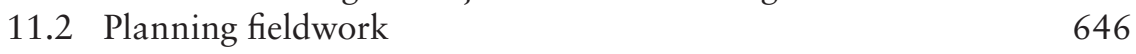

11.3 Types of samples $\quad 648$

11.4 Where to sample 648

11.5 Frequency of site inspection and sampling $\quad 650$

11.6 Personnel and training 654

11.7 Preparative steps and pilot phase 655

11.8 Considerations for downstream analytical procedures $\quad 656$

11.9 Ad hoc sampling following poisoning events 657

11.10 Satellite remote sensing analyses $\quad 658$

11.10.1 Required skills 662

11.10.2 Operational satellites 663

11.10.3 Limitations 664

11.10.4 Frequency of sampling $\quad 664$

11.10.5 Applications for monitoring programmes $\quad 664$

11.10.6 Retrospective assessments 664

11.10.7 Near-real-time monitoring 666

11.10.8 Satellite support of monitoring programmes 666

11.11 Data collection and secure data storage 666

$\begin{array}{ll}\text { References } & 667\end{array}$

\section{INTRODUCTION AND GENERAL CONSIDERATIONS}

As outlined in Chapters 3 and 4, cyanobacteria are likely to be present in any waterbody and hence so are cyanobacterial toxins. The critical issue for the protection of public health is whether concentrations are likely to exceed hazardous levels at points of human exposure. 
For this overall objective, five different types of monitoring serve different specific purposes:

1. Monitoring for risk assessment: Monitoring of waterbodies for the purpose of assessing the risk of cyanobacteria to occur in amounts that may lead to hazardous concentrations does not only target cyanobacteria and cyanotoxins, but also target parameters describing the conditions leading to their proliferation and scum formation (e.g., nutrient concentrations, changes in waterbody stratification or water residence time). Time scales for monitoring in the context of risk assessment are typically once intensively, with periodic checking later on.

2. Monitoring to trigger immediate responses: For example, in the context of an Alert Levels Framework (ALF; see sections 5.1.2 and 5.2.3), it serves to recognise when levels triggering vigilance or alerts are exceeded and corresponding action needs to be taken. This is typically regular (e.g., monthly or weekly), focused on bloom seasons or triggered by exceedance of levels for vigilance or alerts.

3. Monitoring for validation of the control measures in place: It serves to assess whether they are adequate either to prevent cyanobacteria from proliferating to hazardous blooms or to prevent breakthrough of cells and dissolved toxins to the point of water use. For validation, monitoring is intensively done once, when establishing the control system or developing a Water Safety Plan (WSP; see Chapter 6) and is then periodically repeated when the system or WSP is revised. Validation is important for control measures from catchment to consumer, and aspects specific to the catchment, waterbody, site of use and treatment are discussed in the respective Chapters 7-10. While most monitoring for the validation of measures to control concentrations of cyanobacteria and/or cyanotoxins will address their adequacy, it may also include parameters they target, such as visual assessment of erosion in a catchment, nutrient concentrations or temperature profiles.

4. Event-driven monitoring: Monitoring may be triggered by events such as (unexpected) blooms as well as animal deaths or human illness suspected to have been caused by toxic cyanobacteria. The purpose of such event-driven monitoring is usually to identify the cause of the event. Specifically for drinking-water treatment, it may also be to validate efficiency of removal, which can best be done during the event of a heavy bloom. In face of the rapid variability of blooms, particularly of scum situations, sampling as soon as possible during or after the event is key to meaningful data collection: chances for capturing the agents that caused the effects dwindle as time progresses from hours to days. This is most likely to be possible and to provide robust results if sampling is prepared and preplanned, as discussed in 
general in Chapter 15 and specifically for drinking-water treatment in Chapter 10.

5. Monitoring for verification: Confirming that the guideline values are met at the point of exposure involves regular sampling and analysis either of cyanotoxins or of parameters, indicating that cyanobacteria are unlikely to have been present. This is done regularly, possibly limited to the seasons in which they are known to occur.

Note that operational monitoring of control measures is fundamentally different from these five purposes: it serves to ensure that control measures are functioning as intended and that, should one fail, it is possible to respond quickly enough with corrective action to prevent human exposure. Operational monitoring is essential to ensure that systems provide safe water. It typically does not address individual hazards but rather uses a practical, easy-to-measure parameter that shows whether or not a control is functioning. Such parameters range from online recording of turbidity at the outlet of a filter in water treatment to weekly visual inspection of a fence to keep livestock out of a water course. Options for operational monitoring are proposed together with the respective control measures in Chapters 7-10 and are not discussed in the following.

While this chapter focuses on cyanobacteria, cyanotoxins and parameters describing growth conditions favourable for cyanobacteria, it also contains a section on satellite remote sensing. This technology has advanced significantly in the last few decades and is becoming more and more accessible. While remote sensing cannot replace traditional in situ cyanobacteria monitoring and subsequent laboratory analyses, it can be very a useful tool that complements field monitoring in supporting site selection and indicating the frequency of occurrence of blooms of cyanobacteria (or eukaryotic algae).

Water-use systems to be monitored for cyanobacteria and cyanotoxins vary widely, from small ponds to large lakes, from tropical to boreal regions, from small streams to big rivers. No monitoring scheme can be globally applicable to all types of waterbodies, and local conditions have to be taken into account, not least because available resources for a monitoring programme differ substantially (Strobl \& Robillard, 2008; Srivastava et al., 2013). For this reason, this chapter does not propose detailed guidelines, but rather focuses on considerations for designing an appropriate monitoring programme for specific - and often unique - water-use systems.

Bertani et al. (2017) showed that monitoring strategies considerably affect the outcome of cyanobacterial monitoring. Even with expert planning, any monitoring programme inevitably renders data with inherent imprecision. For this reason, one important aspect for long-term monitoring is the continuity of sampling together with detailed documentation. This is crucial for the meaningful interpretation of the data later on - sometimes decades later (e.g., for assessing which changes in the catchment may have changed 
bloom occurrence). Documentation should include not only the sampling procedure and analytical methods, but also the considerations behind any deviation from commonly applied schemes. Further, meaningful data storage in an accessible database is the prerequisite for reliable evaluation of long-term trends.

A basic understanding of limnology is a prerequisite for planning an appropriate monitoring programme for cyanobacterial occurrence in waterbodies; therefore, persons trained in limnology should be consulted in the planning phase, preferably with knowledge of the local waterbodies. Likewise, planning monitoring of schemes for bank filtration or artificial groundwater recharge should involve soil scientists, and planning monitoring schemes for drinking-water treatment needs expertise in treatment technology.

\section{II.I DESIGNING A MONITORING PROGRAMME}

Resources for monitoring can be focused on waterbodies at risk of blooms and, within these waterbodies, on time spans during which they are likely, if the purpose of monitoring is clearly defined. Where potentially toxic cyanobacteria are first recognised as potential risk, it is often possible to use data from past monitoring programmes for a first assessment. It may also be possible to integrate a targeted monitoring into other ongoing programmes.

\section{II.I.I Collecting and analysing existing knowledge}

A first step is to explore which data are already available and whether they allow any estimate of the likelihood of potentially toxigenic cyanobacteria and hence cyanotoxins to occur in the waterbody of interest. In principle, all data on a particular waterbody can be relevant for this purpose, that is, from environmental monitoring programmes and public reports, scientific publications or satellite images (see section 11.4). This may include data on, for example:

- delineation of the catchment, land use and human activities therein (e.g., agricultural practices, waste-water treatment facilities capacity and functioning);

- waterbody morphology, in particular surface area, mean and maximum depth, stratification, water residence time or - for rivers - flow rate;

- types of water use such as drinking-water production, recreational, aquaculture and irrigation;

- location and depth of raw water intake sites (and of alternative locations); 
- location of bathing sites and frequency of seasonal use;

- prevailing wind direction, especially when surface bloom-forming cyanobacteria are abundant (see Chapter 3);

- seasonal dynamics of phytoplankton occurrence and taxonomic composition;

- seasonality and timing of visible cyanobacterial blooms (surface blooms and scums);

- indication of suspected or proven water-related illnesses (human and animal);

- satellite images quantifying total phytoplankton (chlorophyll-a) and cyanobacterial (phycocyanin) biomass, as well as spatial distribution over time and space;

- nutrient concentrations, especially of total phosphorus (TP) and total nitrogen (TN), and their seasonal variation;

- potential major nutrient inputs and possible input fluctuations, for example, seasonality of surface run-off, and possible long-term changes.

Even if the information readily available is only fragmentary, this will support planning and may even allow a first assessment of the likelihood of blooms: specific phytoplankton communities are typical for particular types of waterbodies and seasons (see Chapters 3 and 4). Hence, occurrence of toxic cyanobacterial blooms and related health risks can be foreseen fairly well from basic limnological parameters, even where quantitative phytoplankton data may be lacking or are only rudimentary.

Cyanotoxins are commonly only one among several potential health hazards related to safe water use, and monitoring schemes addressing their occurrence will typically be part of more comprehensive programmes. Their relevance in relation to other hazards in such a programme is best prioritised in the context of overall risk assessment, as discussed in Chapter 6.

If no background data exist, a general limnological screening programme is recommended which may well serve as pilot for a monitoring programme to be subsequently implemented. This would consist of seasonal sampling for basic limnological parameters (e.g., TP, TN, Chl-a, temperature profiles, phytoplankton composition and whether cyanobacteria occur, basic cyanotoxin analysis, e.g., with ELISA) as well as site inspection for general observations (e.g., scum formation, fish and wildlife deaths, water-level fluctuations). The results serve as a starting point to fill some fundamental gaps in data and information, to estimate potential health hazards and potentially to design a full monitoring programme.

If the existing information or the outcomes of such a preliminary screening programme show that for a specific waterbody, a health hazard from cyanotoxins is unlikely (because cyanobacteria hardly occur) or of lower priority relative to other hazards (e.g., pathogens, pesticides or a spill of a hazardous chemical), cyanobacterial monitoring might be reduced to 
observation at low frequency (e.g., once annually during the season in which they are most likely to occur). This would serve to detect changes in the catchment or waterbody that might increase risk, for example, new upstream nutrient loads causing eutrophication or new impoundments changing the mixing regime (see Chapter 4).

\section{II.I.2 Defining the objective of monitoring}

The objective of monitoring, as outlined in points 1-4 in the introduction to this chapter, determines the information needed and time spans and intervals for which it will be necessary (discussed below in section 11.5). The objective also determines where and when samples will be taken in a specific waterbody or along the drinking-water production process as well as chemical and biological analyses to be performed (see Chapters 13 and 14) and the accuracy and sensitivity required. It is therefore very important to clarify the objective(s) early on when planning a programme, both to avoid dispensable efforts (e.g., detection of trace concentrations of cyanotoxins with expensive analytical methods in raw water) and to avoid missing essential information.

\section{II.2 PLANNING FIELDWORK}

Fieldwork, including site inspection, sample collection and, in most programmes, some on-site analyses, largely determines the quality of information obtained from the subsequent laboratory analyses. Fieldwork also causes a significant proportion of the total cost of a cyanobacterial or cyanotoxin monitoring programme. Well-planned and performed sampling is the prerequisite for meaningful results, and most shortcomings in the sampling design cannot be compensated later on: the most accurate and sensitive analytical procedures provide uncertain results if sampling was flawed. A well-designed and implemented fieldwork programme also improves cost-efficiency, that is, for the overall costs of personnel, transport and analytical procedures, by focusing on critical sites and critical periods, as there is little value in spending large amounts of effort on very small risks. This is especially important where the cyanotoxin risk is only one among other health risks from water.

While fieldwork to validate control measures in the catchment or waterbody typically requires a sampling strategy that observes the dynamic changes at a fixed sampling site over time, validating control measures along a drinking-water production process requires a sampling strategy that allows observing changes in a "slug" or "parcel" of water as it passes through the process (see Chapter 10 for details), with a focus on treatment steps that are expected to affect the cyanotoxin concentration. 
For an efficient sampling programme, both sampling schemes need to be coordinated. Validation is most effective if sampling and analyses along the production line are conducted when the raw water at the site of abstraction contains a high concentration of cyanobacteria (possibly including extracellular toxins). Such a situation can be determined using an indicator parameter such as turbidity or fluorimetry (Chapter 5). It is important that sampling and analyses along the production line are launched immediately once a high amount of cyanobacteria is detected in the raw water, in order to have the best chance of determining elimination efficiencies of the individual steps of the treatment train.

The schemes in Figure 11.1 illustrate this, representing two lakes or reservoirs with differing phytoplankton communities (see Chapter 3): the lower scheme shows a lake with a perennial population of cyanobacteria, for example, Planktothrix sp., with a higher base frequency of sampling along the timeline. The upper scheme represents a lake with a strong seasonality of cyanobacteria and cyanotoxin occurrence, for example, the phytoplankton dynamics with a spring bloom of diatoms and dominance of Microcystis sp. in summer. Outside of the cyanobacterial season, the sampling intervals are extended to monthly, while they are reduced to weekly during the blooming season. If the drinking-water production line is to be validated, sampling along the production line is launched once blooms reach a threshold value at the raw water abstraction site, and the production process is then followed through the different steps of the treatment train to the finished drinking-water. If the outcome demonstrates that the treatment train

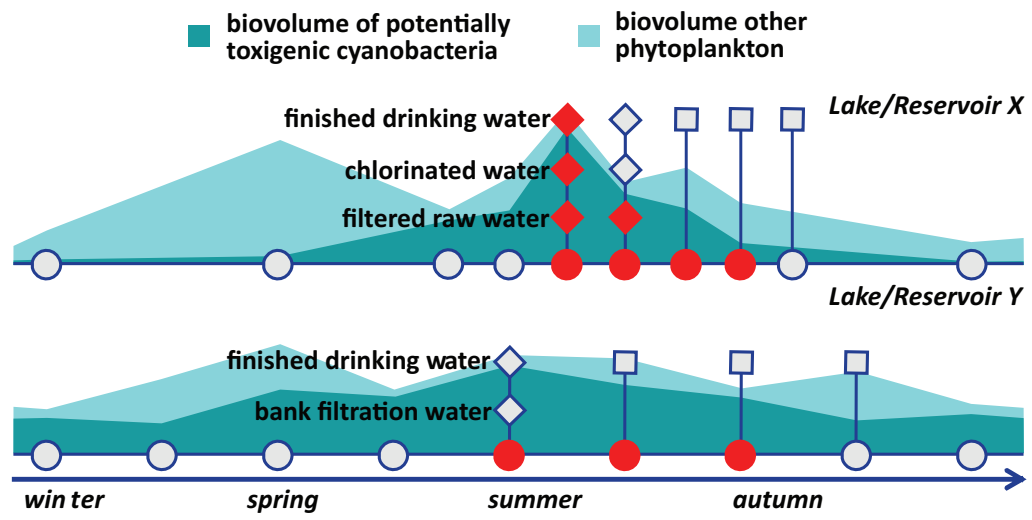

Figure II.I Schematic illustration of sampling strategies and frequencies in two waterbodies used for drinking- water production. Dots: sampling in the waterbody; diamonds: validation sampling along the treatment train ; open symbols: situation above Vigilance Level and below Alert Level I; filled symbols: situation exceeding Alert Level I; open squares: verification monitoring during Alert Level I. Shaded area: occurrence of potentially toxigenic cyanobacteria in relation to other phytoplankton (light area). For details see text. 
effectively controls cyanobacterial cells and toxins, sampling may then be limited to raw and finished water for the rest of the cyanobacterial season until concentrations are below the Vigilance Level of the Alert Levels Framework (ALF) (section 5.1.2). Monitoring may then be suspended until cyanobacteria reappear (possibly not until the following year) in the range of the Vigilance Level. Validation of the drinking-water production line will not be repeated every year if operational monitoring shows control measures to be working effectively and verification monitoring of finished drinkingwater regularly shows concentrations well below the guideline values (see above and Chapter 6). However, as long as concentrations of cyanobacteria and/or cyanotoxins exceed the Vigilance Level or Alert Level 1 in the raw water, it is recommended to include cyanotoxins in a drinking-water utility's routine verification monitoring of finished drinking-water.

\section{II.3 TYPES OF SAMPLES}

For waterbodies, two principally different types of samples may be distinguished: a grab (or spot) sample and an integrated sample. A grab sample is a discrete volume of water taken at a selected location, depth and time. The simplest way to take a grab sample is to scoop water with a wide-mouthed vessel from or near the surface. Subsurface sampling is done with special sampling devices that are also used for integrated sampling. Whereas grab samples are suitable for analysing situations at specific sites (e.g., maximum density of cyanobacteria or cyanotoxins at a bathing site or raw water intake), integrated samples are preferable for assessing the waterbody's average concentrations of substances (e.g., nutrients) or populations of an organism (e.g., the size of a cyanobacterial population).

Integrated samples combine several subsamples from different parts of the waterbody to a combined sample representative for a whole waterbody. These samples are particularly important if the variables to be assessed are unevenly distributed - which is best assumed for most cyanobacterial populations unless a waterbody is well mixed. Integrated sampling may be horizontal, combining samples from different locations, as well as vertical, combining subsamples from multiple depths (for more details, see Chapter 12). The combination of subsamples prior to analysis is often more cost-effective. However, if knowledge of the distribution of parameters is required, each sample can be processed individually.

\section{II.4 WHERE TO SAMPLE}

Ideally, a sample from a waterbody is representative of the water compartment for which information is desired. The water compartment of interest can range from the whole waterbody volume to a mouthful of water swallowed 
by a child. Obviously, this needs to be considered when a monitoring programme is established.

Different waterbody compartments, sampled for different target information and requiring different sampling approaches, include:

- the entire waterbody;

- offtake sites of raw water for drinking-water production;

- sites of recreational activity.

For information to understand bloom development, samples representative of the entire waterbody are necessary. Respective data allow the estimation of, for example, carrying capacity for cyanobacterial biomass, average cyanobacterial abundance, cyanobacterial taxa present and average cyanotoxin concentrations. As outlined above, validating the effects of catchment management or waterbody restoration measures requires integrated sampling (see below and Chapter 12) at moderate frequency (monthly or bimonthly), but for several blooming seasons. Integrated sampling requires more effort than grab sampling because a boat, a water sampler (see Chapter 12), submersible oxygen and temperature probes, and other equipment are needed. Data from integrated samples cannot be used directly for assessing exposure risks at sensitive sites such as drinking-water intakes or bathing sites, where cyanotoxin concentrations can be orders of magnitude higher. The heterogeneous distribution of cyanobacteria in most waterbodies can lead to variability in abundance differing substantially even on narrow spatial scales, horizontally as well as vertically (see Chapters 4 and 9).

Sampling a drinking-water intake can be either in the waterbody at the point immediately before the water enters the drinking-water production system or directly from the raw water pipeline, that is, where it enters the waterworks. However, operators may wish to establish a wider understanding of the occurrence of the specific cyanobacterial population in the waterbody by taking samples representative of the water layer in which they primarily occur, for example, for Planktothrix rubescens in the metalimnion (see Chapter 3), or of a specific bay from which the utility abstracts raw water.

Sampling bathing sites includes shallow waters up to the shoreline and sometimes beyond when cyanobacterial scums have been washed ashore. Cyanobacterial abundance - and hence cyanotoxin concentrations - can fluctuate particularly at near-shore sites by orders of magnitude within days or even hours (see Chapter 4). It is therefore particularly important to clearly define the objective of sampling at respective sites; this determines the number of samples to be taken and the extent to which sampling can simulate the mouthful of water possibly ingested. This could, for example, be the determination of average concentrations for the bathing site up to a certain depth or the estimation of maximally expectable concentrations 
where scums accumulate. Sampling for compliance to the Vigilance Level, as proposed in the Alert Levels Framework (section 5.2.3), may, however, be more effective if it targets representing the entire water volume, as this reflects the overall size of the cyanobacterial population and thus the potential for scum accumulation.

In conclusion, improper sampling may lead to analytically accurate but nonetheless "false" results, which can trigger inappropriate actions or impede necessary steps because the data do not adequately reflect the health hazard.

Samples that cannot be preserved are preferably analysed as soon as possible (i.e., within hours) both for a timely and adequate reaction if results show Vigilance or Alert Levels to be exceeded and for avoiding changes (degradation) in the concentrations of parameters to be analysed.

Good documentation of monitoring is important, and it is valuable to include visual observation (photographs), comments on smells and reports from site users, etc. Where monitoring results of cyanotoxin concentrations or cyanobacterial biomass indicators exceed Alert Level 2 and this leads to restrictions in site use, this can have an immediate economic impact, leading to a high potential for conflicts of interest. Documentation of the rationale for such a decision can then become important. It is also a basis for clear communication between all stakeholders, which is essential for efficient health protection while keeping economic losses low (see Chapter 15).

\section{II.5 FREQUENCY OF SITE INSPECTION AND SAMPLING}

The frequency of site inspection and sampling also needs to be adapted to the objective of the programme. Table 11.1 summarises examples of sampling strategies for these monitoring objectives.

As indicated at the beginning of this chapter, monitoring for risk assessment is an intensive but short-lived exercise that can focus on a small number of sampling campaigns during situations in which blooms are expected to be most likely. It should be repeated at intervals of several years in the context of periodic revision of the management system or the Water Safety Plan, after unexpected blooms or any incident that suggests controls to be insufficient, or if changes in the catchment or other components of the system may have consequences for the adequacy of the control measures in place. A lower number of samples than in the initial campaign may well be sufficient for such repeats.

In contrast, where cyanobacteria are known to occur at potentially hazardous levels, monitoring needs to capture situations in which indicators for toxic cyanobacterial occurrence may exceed predefined thresholds, that is, the Vigilance Levels described in the Alert Levels Frameworks proposed in section 5.1.2 for drinking-water and in section 5.2.3 for recreational 


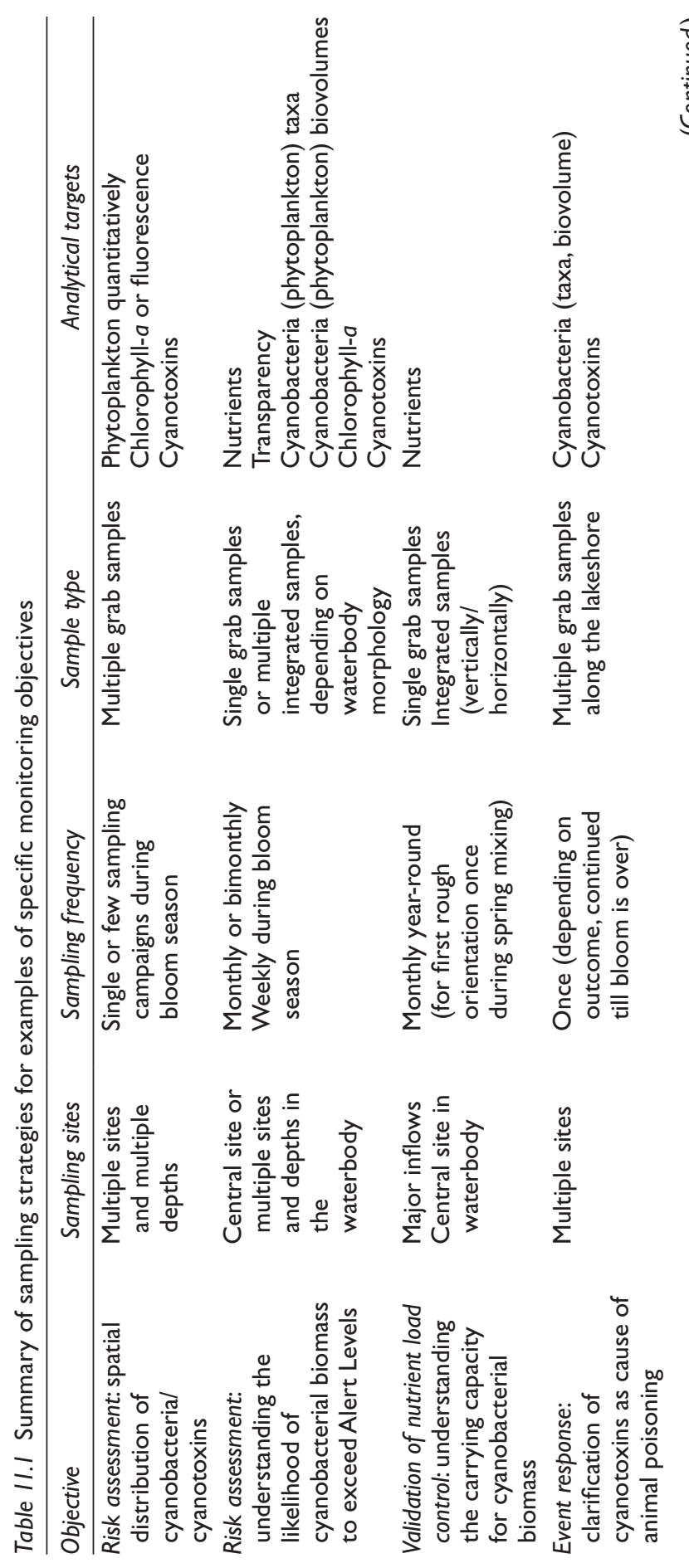




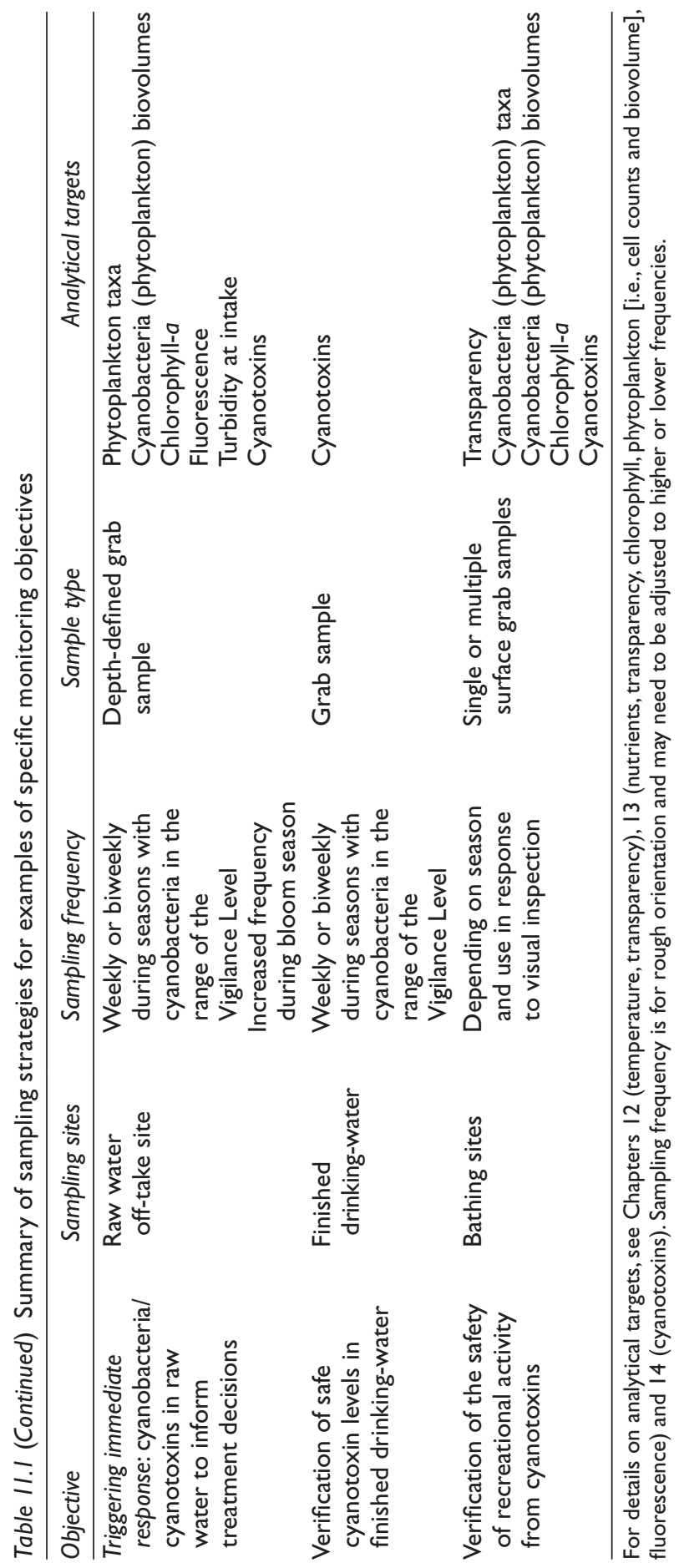


water use. In waterbodies with a pronounced seasonality of cyanobacterial occurrence, this may require an increased frequency of site inspection and sampling during the development of cyanobacterial peak populations, the time of which can be fairly well estimated based on previous data and/ or experiences in other, similar waterbodies in the same climatic region. During peak blooming, information on observations like scum formation at bathing sites is very important (see Chapter 4). If perennial persistence of cyanobacteria cannot be ruled out, drinking-water supply reservoirs may need to be monitored regularly throughout the year for compliance to the Vigilance Level. Persistent cyanobacterial populations can be expected in warm climates and in temperate zones in waterbodies populated by certain taxa like Planktothrix spp. Where monitoring may need to cover the entire year, frequency can be reduced in some season if growth rates are known to then be lower, for example, during the cold season.

Monitoring for compliance to the Vigilance Level is most effective if the time intervals are adapted to bloom occurrence on the basis of a good understanding of the waterbody: if cyanobacteria are known to appear at a certain time or in a certain season, this may initiate monitoring for compliance to Vigilance Levels. For situations exceeding the Vigilance Levels, both Alert Levels Frameworks (ALFs) give guidance for appropriate frequencies of further monitoring. However, once the necessary experience has been developed (typically on the basis of several years of data), it is useful to adapt the frequencies given in the ALFs in sections 5.1 and 5.2 to the bloom development in the given waterbody.

Time scales for monitoring for the purpose of validating the efficacy of control measures may vary widely, depending on the time span for different measures to take effect: for measures in catchment management, it may take years or even decades until reduced nutrient loads lead to reduced cyanobacterial biomass. This is because natural processes in ecosystems can limit or strongly delay responses to newly implemented control measures (Chapters 7 and 8). During this time span, monitoring may be sufficient at low frequency. In contrast, validation of technical measures may be much quicker, that is, in the range of a few days at two to three bloom occasions, for example, for validating whether the variation of raw water intake depth is optimal or the removal efficiency of drinking-water treatment train challenged by a heavy bloom is sufficiently effective (Chapters 7-10). As discussed above, monitoring for validation is conducted once intensively and repeated occasionally (usually after several years) in the context of the revision of the management system or Water Safety Plan. Repeats may also be triggered by changes in the system or incidents questioning whether the measures in place are sufficiently effective.

Monitoring for verification that guideline values for cyanotoxins are met at the points of human exposure is usually well established at regular intervals for microbial indicator organisms and selected chemicals (WHO, 2017). 
While verification monitoring for toxic cyanobacteria should also occur regularly, sampling and analyses may be reduced to seasons in which they are known to occur (see above) or human exposure is likely (e.g., the bathing season). Particularly for the monitoring of bathing sites at which cyanobacteria are known or likely to occur, a high frequency of sampling (i.e., daily or weekly) may be necessary for relatively short periods during the peak bathing season.

\section{II.6 PERSONNEL AND TRAINING}

Properly trained field staff is the backbone of effective sampling and monitoring programmes. Training should include the handling of sampling and measuring devices as well as recognition of visible aspects of cyanobacterial blooms. For smooth and reliable sampling, continuity in staff is highly advantageous. Like with biomass estimation (see Chapter 13), the judgement of a person that knows a system can be very efficient for streamlining the monitoring programme. Experienced field staff should also be encouraged to take additional samples or to make records in case they have the impression that something unusual has occurred. This could be, for example, surface blooms of different colour, dead fish or blooms washed ashore.

Inclusion of the public as active participants in monitoring programmes is gaining acceptance and can contribute significantly to the quantity and quality of information obtained from a monitoring programme. Special interest groups (such as nongovernmental organisations and user associations) as well as concerned local populations in sensitive or affected areas can provide useful information. This is particularly valuable for monitoring sites of recreational activity (see Box 11.1).

\section{BOX II.I: CITIZEN SCIENTISTS AND COMMUNITY PARTICIPATION}

The inclusion of volunteers in the collection of data can significantly support the assessment of the status of diverse environments (for a recent review, see Schröter et al., 2017). In lakes and rivers, it can significantly support the assessment of water quality conditions and cyanobacterial risks. Collected data can range from comparatively simple, such as Secchi depth readings to more complex data like taxonomic composition of phytoplankton communities.

The Secchi Dip-In programme (http://www.secchidipin.org/, sponsored by the North American Lake Management Society [NALMS]) hosts a website with instructions for Secchi depth readings and the option to upload data. The 
data can in turn be accessed for individual lakes. Originally the programme started in the USA but has been adopted in several other countries.

Three coordinated monitoring projects to locate and understand harmful cyanobacteria are conducted by Cyanobacteria Monitoring Collaborative (https://cyanos.org/). The most simple approach is followed by bloomWatch (https://cyanos.org/bloomwatch/) that consists of a free smartphone app and a platform to which digital pictures can be uploaded. The aim of the programme is to track the occurrence of cyanobacterial blooms in waterbodies that are not included in regular, institutional monitoring programmes. The project cyanoScope (https://cyanos.org/cyanoscope/) includes the microscopic observation of plankton samples. A nearly full limnological assessment is the subject of the project cyanoMonitoring (https://cyanos.org/cyanomonitoring/), which requires a more intensive training for interested citizens.

The Centre for Ecology \& Hydrology (UK) provides a similar smartphone app ("Bloomin' Algae") enabling citizens to report algal (surface) blooms (https://www.ceh.ac.uk/algal-blooms/bloomin-algae). A local project primarily targeting public communication, CYANOBs in Potrero de Garay, Argentina, is described in Box 15.2.

Although for all programmes and initiatives the data for individual lakes are highly inconsistent - that is, for some lakes only a few datapoints are available while for others longer time series have been collected - encouraging citizens to collect data could help water managers to extend their knowledge on waterbodies in the region. In spite of reservations voiced about quality control, collected data can, for example, reveal pronounced trends over time (Lottig et al., 20l4) or spatial patterns (Bigham-Stephens et al., 2015) in lake transparency.

Further information concerning planning and performing fieldwork can be found, for example, in Bartram and Ballance (1996), a volume published on behalf of United Nations Environment Programme and the World Health Organization or in United States Geological Survey Guidelines (Graham et al., 2008).

\section{II.7 PREPARATIVE STEPS AND PILOT PHASE}

A period of pilot testing before routine field visits begin helps to ensure that time requirements for inspection and sampling are understood and that activities are planned to make the best use of staff time and other necessary resources (e.g., vehicles). Realistic estimation of travelling time 
between laboratories and sampling sites is important to avoid exceeding tolerable sample storage times prior to analysis. Pilot testing should lead to the development of a detailed inventory and description of sampling sites. If changes in water quality with time are to be interpreted with confidence, samples must be taken consistently from the same locations and/or from other, precisely identified locations. Pilot testing also provides an opportunity for training personnel and familiarisation with the routine.

Coordination with the laboratory responsible for the analyses is an important aspect of preparation. In some cases, the laboratory will be responsible for the preparation of sample containers and chemical additives for sample preservation, and it may also be responsible for the provision and maintenance of equipment for on-site testing (see Chapter 12). Sampling tours also need to be coordinated with downstream analyses to ensure timely sample processing.

\section{II.8 CONSIDERATIONS FOR DOWNSTREAM ANALYTICAL PROCEDURES}

Balancing the costs of the procedures against the depth of information gained is important for an efficient hazard analysis and risk management. The methods for the detection and quantification of nutrients, cyanobacteria and their toxins reviewed in Chapters 13 and 14 range from simple manipulations that can be performed on site to complex techniques that require costly equipment and well-trained experts. The multitude of available methods for cyanotoxin analysis reflects the multitude of situations that demand respective methods. No analytical procedure is superior to others per se - rather, it is the context and purpose that renders an individual method appropriate - or inappropriate. Section 14.2 gives guidance on the scope of the methods for cyanotoxin analyses, and experts in the analytical laboratories will be familiar with most of the advantages and disadvantages discussed there. They should therefore be included when planning a sampling programme.

Laboratory capacity is a further important issue to be addressed in programme design and in pilot testing. It is essential that the workload generated by a sampling expedition is properly managed within the laboratory. Analysts need to know how many samples will be arriving, the approximate time of arrival and the analyses that are to be carried out. Excessive delays before sample processing and analysis may render the sample results invalid (and thereby useless) for the purposes for which they have been collected. Therefore, the timing of sample delivery to the laboratory and the workload management within the laboratory should be coordinated prior to fieldwork.

When planning monitoring programmes (or adjusting them, if necessary), it is important that all participating institutions, companies and laboratories agree on the type and number of samples that eventually are to be 
analysed. This should avoid that samples are taken that are not appropriate for downstream analyses. The following (nonexhaustive) list gives a number of key questions to consider:

- Have the analytical methods to be used been critically evaluated and agreed upon?

- Are the sample types and volumes appropriate for the desired laboratory analyses?

- Is the delay between taking samples and their arrival in the laboratory prone to cause analytical artefacts (e.g., lysis of cells, breakdown of toxins)?

- Could these effects be minimised by (more) appropriate sample handling (see also Chapter 12)?

- Will the samples arrive in the laboratory at a time that allows immediate and appropriate analysis or storage, respectively?

- Have the laboratories been well instructed on sample handling and which analyses are to be performed?

\section{II.9 AD HOC SAMPLING FOLLOWING POISONING EVENTS}

Unexpected events of poisoning particularly of domestic animals continue to be encountered, and comprehensive investigation of such cases can be important to prevent further exposure. It is also relevant to better understand exposure, toxic mechanisms, toxigenic taxa, yet unidentified toxic metabolites and more. For this aim, it is important that laboratories dealing with the analysis of cyanobacteria and their toxins be prepared for ad hoc sampling in case events such as animal deaths or human illness are suspected to be caused by cyanotoxins. The few published reports on such events (e.g., Gugger et al., 2005 and Wood et al., 2017) highlight the importance of having procedures in place to collect samples and information in time, as situations of severe cyanotoxin risks may be only short-lived and an extended delay prior to sampling may make the entire effort futile.

The following list suggests information and items that may help to react adequately to a request of event-triggered sampling:

- Where and when did the poisoning occur?

- Have cyanobacteria/algal mats, scums, pronounced turbidity or smells been observed at the site of poisoning?

- What symptoms have been observed in the casualty?

- Who proposed the diagnosis of cyanobacterial poisoning?

- Where is the casualty now? Is a detailed medical examination planned? Has the examinator been contacted? 
A number of materials should be available ready to use for unexpected poisoning events, preferably in a dedicated "emergency kit":

- sample containers adequate for water, algal mats, macrophytes, phytoplankton microscopy etc;

- sample containers for animal tissue samples, stomach contents;

- contact details of physicians and veterinarians that may be involved or consulted;

- storage space in freezers and fridges, a cooling box for transport.

Chapter 15 gives further guidance for responding to events and emergencies.

\title{
II.I0 SATELLITE REMOTE SENSING ANALYSES
}

Remote sensing can serve as a starting point to plan a monitoring programme, for example, by identifying the season of cyanobacterial blooms or locating sites of biomass accumulation. Once a monitoring programme is in place, remote sensing can serve to verify the validity of sampling points with respect to their representativeness of the monitored waterbody. Previous satellite data can provide historical assessments. As shown by the example in Box 11.2, remote sensing further provides a rather low-cost opportunity to intensify monitoring in time and space, particularly in areas for which a large number of sampling points and/or frequent visits for sampling would be necessary.

\section{BOX II.2: REMOTE SENSING OF CYANOBACTERIA: THE CASE STUDY OF SALTO GRANDE RESERVOIR}

\author{
Andrea A. Drozd
}

Salto Grande reservoir, constructed in 1979, impounds the Uruguay River which divides Argentina and Uruguay. It is visited by thousands of tourists during summer in spite of recurrent and often heavy cyanobacterial blooms (O'Farrell et al., 2012; Bordet et al., 2017) with cyanobacterial cell density and microcystin concentrations frequently surpassing the recreational guideline levels given by $\mathrm{WHO}$, and one case of severe liver damage was described after a young jet skier had spent many hours in the bloom (see section 5.2 and Giannuzzi et al., 20II). The reservoir's large area of $750 \mathrm{~km}^{2}$ and its dendritic morphology with lateral arms renders sufficiently frequent and comprehensive sampling impossible. Since $201 \mathrm{I}$, monitoring cyanobacteria blooms 
and water quality is being complemented with a remote sensing monitoring programme developed by the "Comisión Administradora del Río Uruguay" (CARU, 2016; 2017). It targets quantifying chlorophyll-a concentrations by satellite data at a scale of $1: 50000$.

From 2012 to 2016 , 10 field campaigns provided a basis for this by characterising spectral signatures of dam water under different conditions using samples from multiple sites for laboratory analyses (by Comisión Mixta de Salto Grande) of chlorophyll-a concentration, phytoplankton composition and abundance as well as turbidity, together with in situ hyperspectral signatures obtained by a hyperspectral radiometer (ASD Field Spec provided by the Comisión Nacional de Actividades Espaciales) in order to describe how suspended matter, chlorophyll-a concentration, phytoplankton composition and cyanobacteria cell density influence the spectral patterns. These spectral signatures were then used to build band algorithms for sensors of different satellites, that is, Landsat 7-8 and Sentinel 2, SPOT HRVIR, relating spectral pattern characteristics to concentrations of chlorophyll- $a$ and cyanobacterial cell density (Drozd et al., 2020).

A first result was the spectral discrimination of phytoplankton communities. With Landsat and Sentinel 2, dominance of dinoflagellates showed a dark-blue colour (Figure I, Panel A), and the absorption of the green spectral range increased with their cell density; in contrast, cyanobacteria showed an inverse response: the greater their biomass, the lower the absorption in the green spectral range, leading to a bright green colour when cyanobacteria dominated (Figure I, Panel B). A second result was the relationship between chlorophyll-a concentration and a band index algorithm $\left(R^{2}>0.77\right)$, allowing the monitoring of phytoplankton intensity and distribution as the basis for deciding on priority sites for field sampling, indicating the hotspots where blooms originated as well as beaches with potential health risks (Figure I, Panel C).

As the next step, for situations with dominance of cyanobacteria, a cell density algorithm was developed, allowing estimation by satellite data. CARU has established Alert Levels for recreational use of waterbodies using observed colour patterns of the water which coincide with cyanobacteria cell density and toxin concentrations. This cyanobacterial algorithm is able to detect average densities of 200 cells $/ \mathrm{mL}$ and hence to map CARU's Alert Levels (Figure I, Panel D).

Since 2012, chlorophyll- $a$ and cyanobacterial remote sensing proved a helpful tool for a synoptic understanding of spatiotemporal dynamics of 
blooms in Salto Grande and for providing an estimate of cell densities. In summertime, when rain is scarce, satellite data can be obtained at intervals of 3-5 days, enabling low-cost monitoring of phytoplankton communities, short-term reports and warnings as well as more effectively targeted sampling programmes. For more information, see www.caru.org.uy and www. saltogrande.org

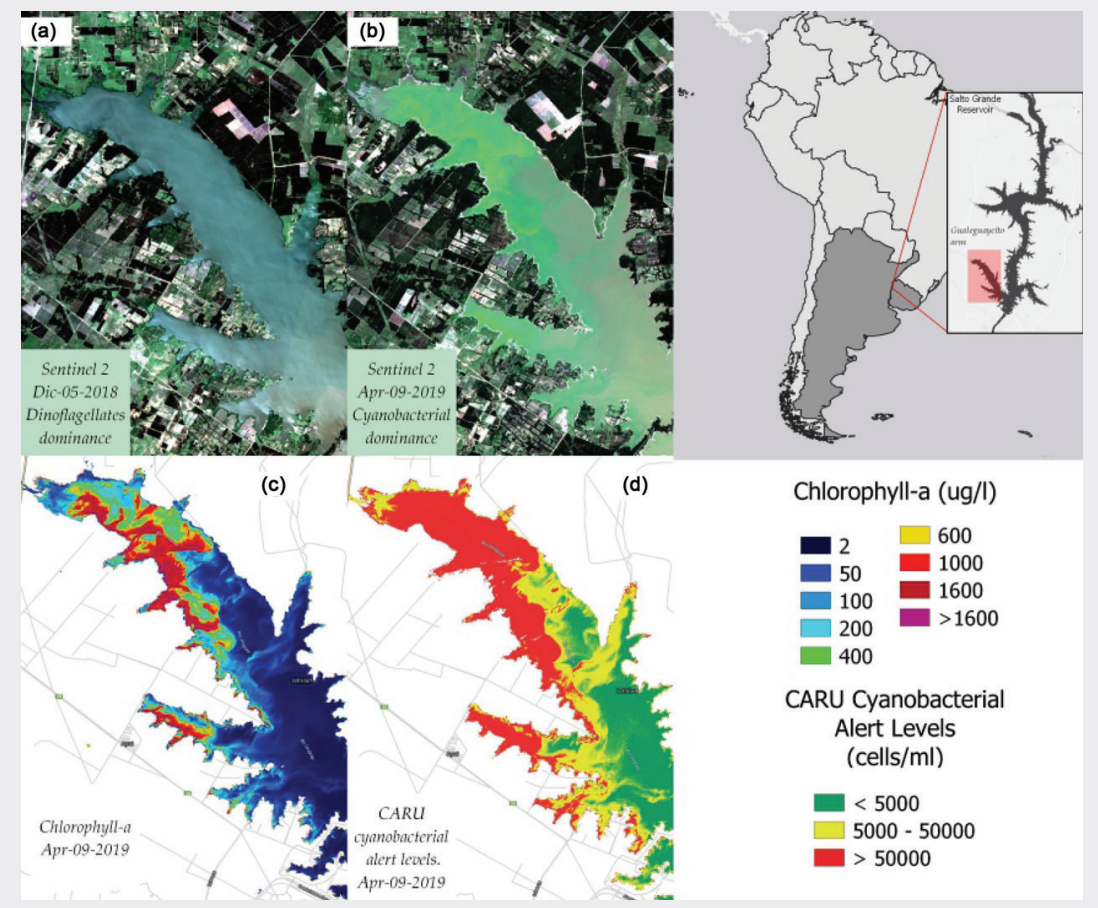

Figure I Salto Grande Reservoir, Gualeguaycito arm. Images obtained with Sentinel 2. Panel A: 05 December 2018 with dominance of dinoflagellates; Panel B: 9 April 2019 with cyanobacterial blooms. Panel C: Chlorophyll-a range estimation by Sentinel 2 on 9 April 2019. Panel D: Cyanobacterial cell density ranges estimated by Sentinel 2 on 9 April 2019, reflecting CARU cyanobacterial Alert Levels.

Available remote sensing systems are generally based on satellite images and may also be available from platforms such as drones and airplanes. The focus here will be satellites for monitoring water quality, instruments that orbit Earth in space. In contrast to drones and airplanes, data from many government operational satellite sensors are available free of charge. 
Satellites can contain multiple sensors that provide a birds-eye view of the Earth's surface. Satellite sensors designed for water quality measures are typically passive sensors, which means they detect changes in sunlight reflected off the water surface. When light interacts with the water environment, it can either be absorbed or scattered in the water column (Figure 11.2). Dissolved and particulate matter in the water column absorb and scatter light differently across the wavelengths of the visible light spectrum. Changes in the visible light spectrum by materials present in the water column, like pigments in phytoplankton and cyanobacteria, can be quantified by these satellite sensors. Firstly, the sensor detects the spectral changes and then validated mathematical algorithms quantify concentrations of these water column materials. Algorithms are successful at quantifying both phytoplankton (chlorophyll-a) and cyanobacteria biomass concentrations (Figure 11.3). Satellite sensor technologies typically follow a transition pathway starting with research and development of theoretical

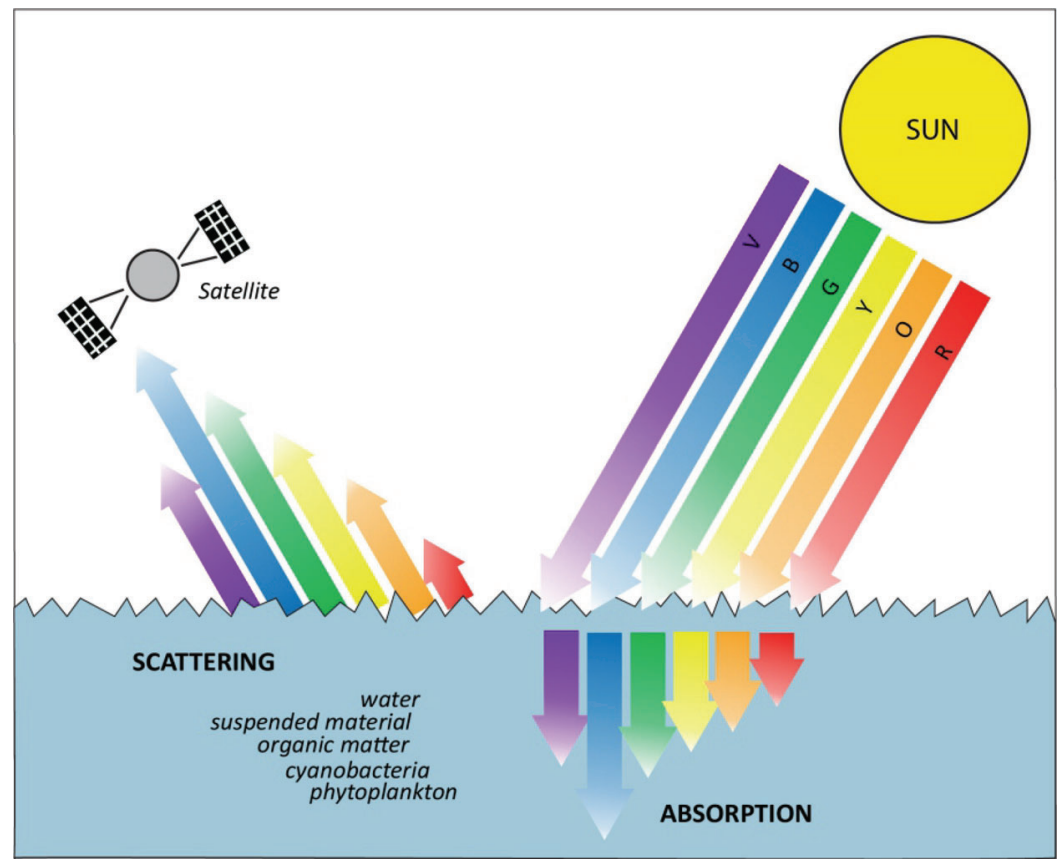

Figure II.2 Conceptual diagram of how a typical satellite sensor detects water quality changes from sunlight reflected off the water surface. Light can either be absorbed or be scattered through interaction with water, phytoplankton, cyanobacteria, organic matter and suspended material across wavelengths of the visible spectrum. Changes in the visible light spectrum from scattering and absorption can be quantified with algorithms to derive measures of, for example, cyanobacteria biomass. 


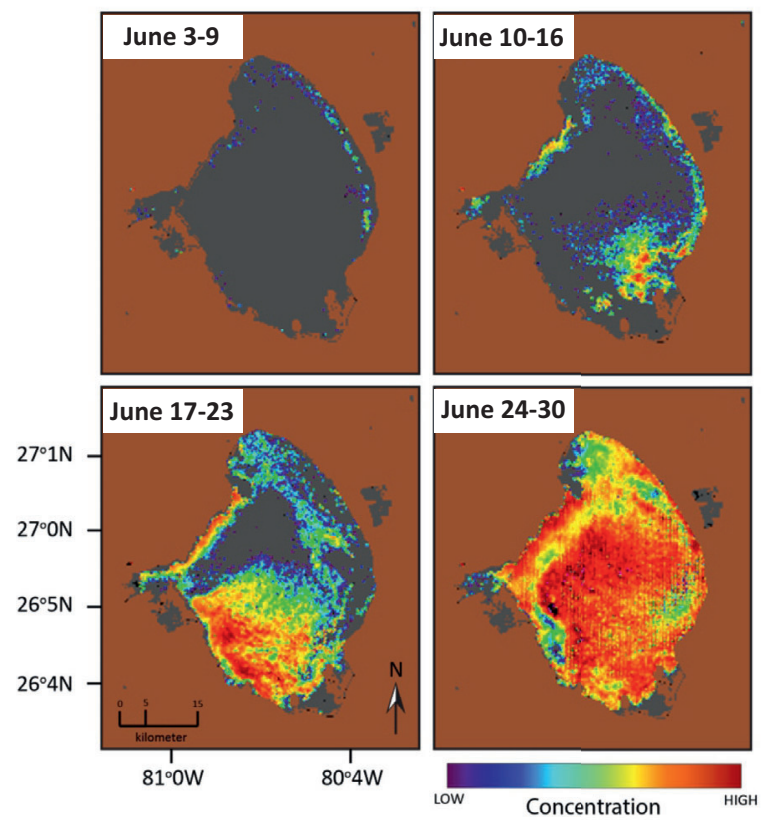

Figure II.3 Exemplary time series of the Sentinel-3 OLCl satellite images of Lake Okeechobee, Florida, that can quantify the temporal and spatial changes of cyanobacteria biomass within a waterbody. Brown pixels are land masks; black and dark-grey pixels indicate no data (i.e., cloud cover) and below the algorithm detection limit, respectively. Coloured pixels indicate biomass of cyanobacteria from high (red) to low (purple).

and engineering proof-of-concept sensors, and progress towards method development, like water quality algorithms. Eventually, satellite technology and developed methods transition towards operational satellites for the incorporation of data by users, like water quality management. A thorough review of satellite technology and considerations for water quality management is provided in the International Ocean Colour Coordinating Group, Earth Observations in Support of Global Water Quality Monitoring Report (IOCCG, 2018).

\section{II.I0.I Required skills}

Typically, new users of satellite data will require computer hardware and expertise to enable adequate data processing and interpretation. Using satellite technology for water quality monitoring and assessment would likely require some new staff training, new software applications and at least a 
basic understanding of the fundamentals behind remote sensing technology (Schaeffer et al., 2013). Training should include understanding operational satellite platforms, data acquisition, data extraction, quality control and limitations of the applied methods such as algorithm accuracy, uncertainties, interferences and data quality. New software may include free and open source programs such as the National Aeronautics and Space Administration's (NASA) Sea-Viewing Wide Field-of-View Sensor (SeaWiFS) Data Analysis System (SeaDAS), European Space Agency's (ESA) Sentinel Application Platform (SNAP) or R computer language. Other software may include Geographic Information System (GIS) packages, web-based portals and various for purchase software options. Basic information from picture formats (JPEG, TIFF, etc.), without georeference, may also be informative.

As shown by the example in Box 11.2, it is important to validate the satellite-derived results with field measurements. It is also important to report accuracy or error estimates for the specific waterbodies.

\section{II.I0.2 Operational satellites}

Current and future operational satellite sensors all have some limited ability to resolve the required geophysical variables but with significant trade-offs among spectral, spatial or temporal resolution (Mouw et al., 2015; Palmer et al., 2015). Here we only mention open-access operational satellites with the highest potential to inform management decisions for inland waters which exist at the time of the publication of this book. These operational satellites generally fall into two categories: (1) medium-resolution ocean colour sensors and (2) higher-resolution land imagers. The medium-resolution ocean colour sensors may include ESA's Ocean and Land Colour Instrument (OLCI) on the Sentinel-3 (3A launched 2016 and 3B launched in 2018) satellites. Historical data could be retrieved from the MEdium Resolution Imaging Spectrometer (MERIS) on the Envisat satellite from 2002 to 2012. The OLCI and MERIS sensors provide adequate spectral bands for inland water derivation of water quality parameters, with a typical revisit time of 2-3 days, but have spatial resolution ( $300 \mathrm{~m}$ pixel size) limits. Thus, they are useful for providing observations in larger lakes and reservoirs. The higher-resolution land imagers include the Multi-Spectral Instrument (MSI) on the Sentinel-2 (2A launched 2015, 2B launched in 2017) satellites and Landsat series satellites provide the best spatial resolution for inland waters but are at a disadvantage when it comes to spectral resolution, signal-to-noise ratio, and, to some extent, temporal coverage. Only by combining the observations from Landsat missions or Sentinel-2 missions would a temporal revisit of every 8 days and 5 days be possible, respectively. The satellite revisit time is defined as the time between measurements of the same location on the surface of the Earth. 


\section{II.I0.3 Limitations}

Satellite data may be used to infer surface bloom locations throughout the waterbody, albeit with some technological limits. For example, mixed land and water pixels, and bottom interference may confound the derivation of remote sensing results along the lakeshore. All satellite algorithms detect only near-surface concentrations, and the red and near-infrared part of the spectrum provide information from only the upper few metres of the water column. Atmospheric interference, cloud cover and ice formation limit the usability of satellite images at different rates, depending on the climate of the location. Overall waterbody size and optical complexity impact the application of satellites based on the native pixel resolution of the sensor and processing steps, respectively. There is no optical signal that can be detected by satellites to directly measure cyanotoxin concentrations.

\section{II.I0.4 Frequency of sampling}

Satellite remote sensing presents a cost and time-effective approach complimentary to field-based cyanobacterial monitoring efforts for a more comprehensive assessment of inland waters. Remote sensing can provide water quality data with frequent revisit times for many lakes. These operational satellites provide daily, 2-3 day, weekly, monthly and seasonal assessments of water quality data. The near-real-time availability of water quality data from current satellites makes it possible to integrate such data into early warning systems to protect human health and ecosystems.

\section{II.10.5 Applications for monitoring programmes}

Historical satellite records may be used to contextualise background monitoring to identify the potential for cyanobacteria occurrence problems in waterbodies and the typical timing, location and extent of the bloom at local and regional scales. Near-real-time satellite records may be used for cyanobacteria monitoring to quantify abundance in recreational and drinking waterbodies.

\section{II.10.6 Retrospective assessments}

Satellite remote sensing may be used to quantify the spatial extent of the surface area covered by a cyanobacterial bloom (Urquhart et al., 2017). Relevant statistical tests and time-series analyses may be used to identify trends in satellite-derived extent of surface area covered by cyanobacteria. Trend analysis for surface area extent may be subdivided into categorical thresholds desired by the user, based on cyanobacteria concentration or chlorophyll- $a$, to help water managers effectively distribute resources to monitor and manage waters. Scalable assessments may permit the development of management objectives 
over different temporal periods and spatial scales. Improved multiscale assessment capability is desirable so that comparisons of condition may occur across local, regional and national scales to more adequately evaluate regional water quality, biological integrity and response to management actions.

The frequency of observed cyanobacteria (Figure 11.4) may be calculated as the fraction of total observations for which cyanobacteria biomass exceeded a specified threshold, for example, a Vigilance or Alert Level (sections 5.1.2 and 5.2.3; Clark et al., 2017). Values are summed for each pixel and divided by the total number of valid observations (i.e., those not flagged for clouds, land, mixed land water and lack of data). Finally, the magnitude of cyanobacteria biomass may be calculated based on the spatiotemporal mean of the biomass for a particular period of time such as a season or year. The spatial extent, temporal frequency and magnitude can all be used to rank waters in order of importance to prioritise management resources (Mishra et al, 2019).

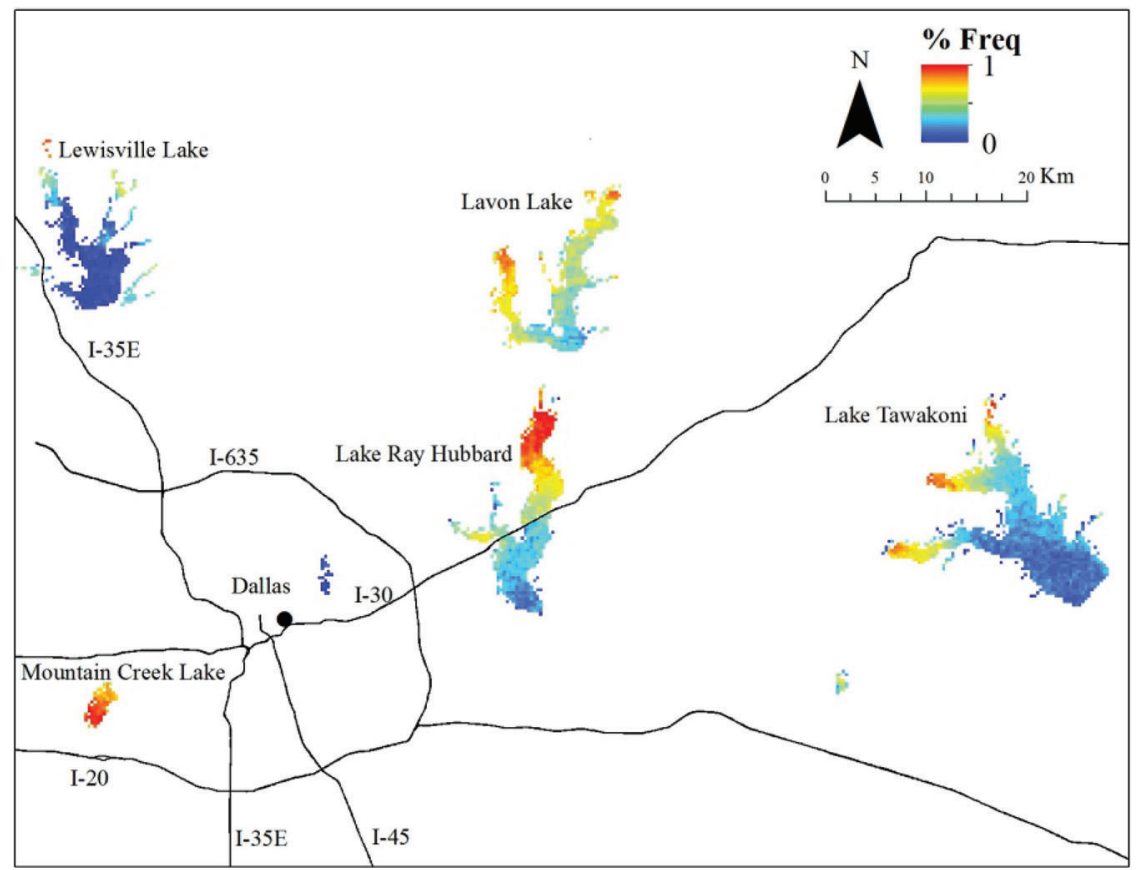

Figure II.4 A demonstration of how ESA's Sentinel-3 OLCl satellite data can be used for a quantitative retrospective assessment across lakes near Dallas, Texas, USA. Here, the frequency of detected cyanobacteria biomass is calculated as the fraction of total observations throughout 2017. A value of I indicates cyanobacteria had a $100 \%$ frequency of occurrence in valid observations through 2017, and a value of 0 indicates cyanobacteria were not detected. 


\section{II.I0.7 Near-real-time monitoring}

Satellites provide a constant birds-eye view of the Earth's surface and can be used to identify changes in the environment across geopolitical boundaries by providing updated images the same day the data is acquired. This information may be used to identify events and locations during days of data acquisition, or at weekly, monthly and seasonal intervals. Management decisions such as updating recreational beach notices or modifying drinking-water treatment methods may benefit from access to near-real-time satellite-derived occurrence information.

\section{II.I0.8 Satellite support of monitoring programmes}

The presence of cyanobacteria or chlorophyll- $a$ biomass estimated from satellites may be used as a first-line indicator of potential ecological and human health risk that can be used to prioritise waterbodies requiring further evaluation for parameters such as visual inspection, laboratory assessment of cyanobacteria taxon composition and biomass, and assessment of cyanotoxin concentrations. Satellites have the potential to support monitoring efforts across broad geographic extents and provide improved temporal and spatial coverage at larger scales. When coupled with field-based observations, satellite data provide a more comprehensive tool to monitor, assess and detect changes in the environment. The science required for a more precise interpretation of satellite remote sensing of water quality such as in-water algorithms, atmospheric corrections and land adjacency effects will continue to mature over the coming decades. Significant progress has already been demonstrated in deriving cyanobacteria and chlorophyll- $a$ data from inland and estuarine waters using satellite sensors.

More information on satellite remote sensing, including training webinars, and access to a community of practice are available from the Group on Earth Observations AquaWatch website (https://www.geoaquawatch.org/).

\section{II.II DATA COLLECTION AND SECURE DATA STORAGE}

Consistent time series are very valuable to observe long-term changes in a waterbody's condition. To make use of time series, the data need to be collected in a repository that allows reliable access, preferably in a centralised database. A database is best curated by a single person or a few responsible persons/institutions, and any data entered in a database should be as uniform as possible. In particular, units for individual values have to be standardised - confusion may arise from different units when they are not clearly disclosed, that is, concentrations in $\mu \mathrm{g} / \mathrm{L}$ or $\mathrm{ng} / \mathrm{L}$ or $\mathrm{ng} / \mathrm{mL}$. This is especially important when data are compiled from several individual 
institutions and laboratories. Although this is in theory self-evident, the lack of uniformity of data is a constant source of hassle, particularly for supra-regional or supra-national data analysis and interpretation.

Monitoring programmes tend to require an adaption to changing circumstances as time progresses. This could be changes in sampling frequency, sampling point locations or analytical methods, for example. Any modification needs to be well documented to ensure traceability of the data. So do the analytical methods used. In addition to the laboratory quality assurance system implemented for sampling and analyses, a plausibility check of data prior to their final storage helps identify trivial errors that creep in, for example, due to misplacements of decimals. Again trivial, but often missed is that a timely check allows for questions back to those performing sampling and analyses in case data do not appear plausible, and this may well lead to further information explaining unusual data which is important to include in the documentation.

\section{REFERENCES}

Bartram J, Ballance R, editors (1996). Water quality monitoring: a practical guide to the design and implementation of freshwater quality studies and monitoring programmes. London: F \& FN Spoon on behalf of United Nations Environment Programme and the World Health Organization:383 pp.

Bertani I, Steger CE, Obenour DR, Fahnenstiel GL, Bridgeman TB, Johengen TH et al. (2017). Tracking cyanobacteria blooms: Do different monitoring approaches tell the same story? Sci Tot Environ. 575:294-308.

Bigham-Stephens DL, Carlson RE, Horsburgh CA, Hoyer MV, Bachmann RW, Canfield DEJ (2015). Regional distribution of Secchi disk transparency in waters of the United States. Lake Reserv Manage. 31:55-63.

Bordet F, Fontanarrosa MS, O'farrell I (2017). Influence of light and mixing regime on bloom-forming phytoplankton in a subtropical reservoir. River Res Appl. 33:1315-1326.

CARU (2016). Estudio de la calidad del agua del Río Uruguay. Bienio 2013-2014. Paysandú: Comisión Administradora del Río Uruguay.

CARU (2017). Programa de vigilancias de playas del Río Uruguay. Paysandú: Comisión Administradora del Río Uruguay. http://www.caru.org.uy/ web/2017/12/programa-de-vigilancia-de-playas-del-rio-uruguay/.

Clark JM, Schaeffer BA, Darling JA, Urquhart EA, Johnston JM, Ignatius AR et al. (2017). Satellite monitoring of cyanobacterial harmful algal bloom frequency in recreational waters and drinking water sources. Ecol Indicators. 80:84-95.

Drozd A, de Tezanos Pinto P, Fernandez V, Bazzalo M, Bordet F, Ibañez G (2020). Hyperspectral remote sensing monitoring of cyanobacteria blooms in a large South American reservoir: high-and medium-spatial resolution satellite algorithm simulation. Mar Freshwater Res. 71:593-605.

Giannuzzi L, Sedan D, Echenique R, Andrinolo D (2011). An acute case of intoxication with cyanobacteria and cyanotoxins in recreational water in Salto Grande Dam, Argentina. Mar Drugs. 9:2164-2175. 
Graham J, Loftin A, Zeigler A, Meyer M (2008). Cyanobacteria in lakes and reservoirs-toxin and taste-and-odor sampling guidelines (version 1.0). Reston (VA): US Geological Survey.

Gugger MF, Lenoir S, Berger C, Ledreux A, Druart JC, Humbert JF et al. (2005). First report in a river in France of the benthic cyanobacterium Phormidium favosum producing anatoxin-a associated with dog neurotoxicosis. Toxicon. 45:919-928.

IOCCG (2018). Earth observations in support of global water quality monitoring. Dartmouth: International Ocean Colour Coordinating Group. www.ioccg.org. Lottig NR, Wagner T, Henry EN, Cheruvelil KS, Webster KE, Downing JA et al. (2014). Long-term citizen-collected data reveal geographical patterns and temporal trends in lake water clarity. PloS One. 9:e95769.

Mishra S, Stumpf RP, Schaeffer BA, Werdell PJ, Loftin KA, Meredith A (2019) Measurement of cyanobacterial bloom magnitude using satellite remote sensing. Sci Rep 9:1-17

Mouw CB, Greb S, Aurin D, DiGiacomo PM, Lee Z, Twardowski M et al. (2015). Aquatic color radiometry remote sensing of coastal and inland waters: challenges and recommendations for future satellite missions. Rem Sens Environ. 160:15-30.

O'Farrell I, Bordet F, Chaparro G (2012). Bloom forming cyanobacterial complexes co-occurring in a subtropical large reservoir: validation of dominant eco-strategies. In: Salmaso N, Naselli-Flores L, Cerasino L et al., editors: Phytoplankton responses to human impacts at different scales. Cham: Springer Nature:175-190.

Palmer SC, Kutser T, Hunter PD (2015). Remote sensing of inland waters: challenges, progress and future directions. Remote Sens Environ. 157:1-8.

Schaeffer BA, Schaeffer KG, Keith D, Lunetta RS, Conmy R, Gould RW (2013). Barriers to adopting satellite remote sensing for water quality management. Int J Remote Sens. 34:7534-7544.

Schröter M, Kraemer R, Mantel M, Kabisch N, Hecker S, Richter A et al. (2017). Citizen science for assessing ecosystem services: status, challenges and opportunities. Ecosyst Serv. 28:80-94.

Srivastava A, Singh S, Ahn C-Y, Oh H-M, Asthana RK (2013). Monitoring approaches for a toxic cyanobacterial bloom. Environ Sci Technol. 47:8999-9013.

Strobl RO, Robillard PD (2008). Network design for water quality monitoring of surface freshwaters: a review. J Environ Manage. 87:639-648.

Urquhart EA, Schaeffer BA, Stumpf RP, Loftin KA, Werdell PJ (2017). A method for examining temporal changes in cyanobacterial harmful algal bloom spatial extent using satellite remote sensing. Harmful Algae. 67:144-152.

WHO (2017). Guidelines for drinking-water quality, fourth edition, incorporating the 1st addendum. Geneva: World Health Organization:631 pp. https://www. who.int/publications/i/item/9789241549950

Wood SA, Puddick J, Fleming RC, Heussner AH (2017). Detection of anatoxinproducing Phormidium in a New Zealand farm pond and an associated dog death. New Zeal J Botany. 55:36-46. 